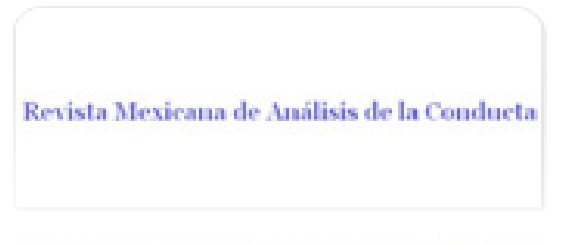

Revista Mexicana de Análisis de la Conducta ISSN: 0185-4534

editora@rmac-mx.org

Sociedad Mexicana de Análisis de la Conducta México

TORRES, CARLOS; VILLAMIL, WILCEN; CALLEROS, KARLA EFECTOS DE LA DISMINUCIÓN DE LA PROBABILIDAD Y LA DISPONIBILIDAD DE REFORZAMIENTO EN PROGRAMAS TEMPORALES DE DISTINTA DURACIÓN

Revista Mexicana de Análisis de la Conducta, vol. 35, septiembre, 2009, pp. 87-100

Sociedad Mexicana de Análisis de la Conducta

Guadalajara, México

Disponible en: http://www.redalyc.org/articulo.oa?id=59312304007

Cómo citar el artículo

- Número completo

- Más información del artículo

- Página de la revista en redalyc.org

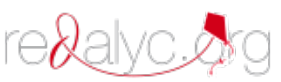

Sistema de Información Científica

Red de Revistas Científicas de América Latina, el Caribe, España y Portugal Proyecto académico sin fines de lucro, desarrollado bajo la iniciativa de acceso abierto 


\title{
EFECTOS DE LA DISMINUCIÓN DE LA PROBABILIDAD Y LA DISPONIBILIDAD DE REFORZAMIENTO EN PROGRAMAS TEMPORALES DE DISTINTA DURACIÓN
}

\author{
EFFECTS OF DECREASING THE PROBABILITY AND \\ THE AVAILABILITY OF REINFORCEMENT IN DIFFERENT LENGTH \\ TEMPORAL SCHEDULES \\ CARLOS TORRES, WILCEN VILLAMIL Y KARLA CALLEROS ${ }^{1}$ \\ UNIVERSIDAD DE GUADALAJARA \\ CENTRO DE ESTUDIOS E INVESTIGACIONES EN COMPORTAMIENTO
}

\section{RESUMEN}

Se desarrolló un estudio para evaluar los efectos de manipular la probabilidad y la disponibilidad de reforzamiento utilizando programas definidos temporalmente. Doce ratas albino Wistar fueron divididas en tres grupos. Cada uno de los grupos se expuso a tres fases experimentales en las que se varió la probabilidad de reforzamiento en los siguientes valores: $1.0,0.5$ y 0.1 . En cada una de las fases experimentales se establecieron cuatro bloques en los que se manipuló el valor de la disponibilidad de reforzamiento $(\bar{T}=1.0,0.5$, $0.3,0.1$ ). Los resultados sugieren que la frecuencia de respuesta desciende sistemáticamente conforme disminuye la probabilidad de reforzamiento; sin embargo, la dirección del efecto parece depender de la duración absoluta de T. Por otro lado, los datos muestran una función descendente en el porcentaje de entregas de agua obtenidas asociadas a los decrementos de los valores

1. El primer autor fue el responsable de coordinar los trabajos de laboratorio de los autores 2 y 3 , los cuales a su vez se encargaron del análisis de datos. Agradecemos los comentarios del Dr. Carlos Flores y el Dr. Mario Serrano respecto de los resultados obtenidos en este estudio. La correspondencia sobre este artículo deberá dirigirse al Dr. Carlos Torres, al correo electrónico: jtorres@ cencar.udg.mx.

Recibido: 14 de abril de 2009. Revisado: 6 de mayo de 2009. Aceptado: $1^{\circ}$ de julio de 2009. 
de disponibilidad de entrega de agua. Estos resultados son examinados en términos de las propiedades de los programas definidos temporalmente y de la distribución local de la respuesta y la entrega de agua.

Palabras clave: probabilidad de reforzamiento, disponibilidad de reforzamiento, programas temporales, duración de ciclo.

\begin{abstract}
One study was conducted to evaluate the effect of varying the reinforcement probability and availability in a temporally-defined schedule. Twelve male albino Wistar rats were divided into three groups. Each group were exposed to a three experimental phases with reinforcement probability $(p)$ set at 1.0, 0.5 and 0.1 . Into every experimental phase, there were set four blocks of different reinforcement availability values $(\bar{T}=1.0,0.5,0.3,0.1)$. The results suggest that response frequency decreased systematically with overall reinforcement probability. However, this effect seems to depend on T cycle duration. On the other hand, data shows a decrement function in percentages of water deliveries associated to decrements in water delivery availability $(\check{T})$. These results are examined in relation to properties of temporally-defined schedules and the local distribution of water deliveries and responses.

Keywords: probability of reinforcement, availability of reinforcement, temporal schedules, cycle duration.
\end{abstract}

La concepción del estímulo y la respuesta como clases genéricas (Skinner, 1938) permitió que todas las instancias de respuesta fueran vistas como eventos idénticos en la medida en que cumplieran con los requisitos definitorios de la clase. Dado esto, la operación de reforzamiento en los estudios de condicionamiento operante, es decir, la operación de presentación de un estímulo reforzante en relación con una respuesta, implicaba la identificación tanto de la instancia particular a la cual el reforzador debía seguir, como de la clase de evento consecuente que afecta la conducta en términos de sus propiedades funcionales. Las dos únicas posibilidades para la identificación de la instancia de respuesta particular en un flujo continuo de emisiones $(u$ ocurrencias) eran: a) su posición en el tiempo considerada a partir de un cero arbitrario; y b) su posición ordinal contada también a partir de un cero arbitrario (Schoenfeld, Cumming \& Hearst, 1956).

Los programas de intervalo y de razón que resultaron de la aplicación de estas dos posibilidades, así como sus distintas combinaciones y variedades, constituyeron las reglas para identificar instancias de respuesta a re- 
forzar. Además, el carácter sistemático de los efectos de los programas de reforzamiento sobre la tasa de respuesta y el patrón de ejecución, sirvieron de fundamento a la suposición de que las ejecuciones resultantes eran determinadas por la acción de variables distintas. Tales variables fueron las prescritas por los propios programas y, como consecuencia, las diferencias en las ejecuciones fueron explicadas satisfactoriamente por referencia a los programas mismos (Ferster \& Skinner, 1957).

Los programas de reforzamiento surgieron, por lo tanto, como instrumentos para analizar las relaciones entre las operaciones de reforzamiento y los cambios en la fuerza de la respuesta. Sin embargo, no existen informes en la literatura del condicionamiento operante acerca de las ejecuciones obtenidas por programas en los cuales la probabilidad de reforzamiento sea diferente de 1.0 y 0.0 . Esto puede deberse a que en los programas tradicionales de reforzamiento, aunque se pude manipular de manera independiente la frecuencia y la distribución temporal del reforzador, la probabilidad de reforzamiento se mantiene constante en el valor de 1.0. Es posible que las únicas excepciones hayan sido los programas de porcentaje (Neuringer \& Chung, 1967) y los programas de omisión (Staddon \& Innis, 1969), en los que los reforzadores programados pueden no presentarse. De hecho, como lo comentan Ribes, Zepeda, Arenas y Mayoral (2007), los programas de intervalo y de razón son variaciones del programa de reforzamiento continuo (Rfc): Razón Fija $1\left(\mathrm{RF}_{1}\right)$ o Intervalo Fijo $0\left(\mathrm{IF}_{0}\right)$.

Los programas temporales (Schoenfeld \& Cole, 1972; Schoenfeld et al., 1956) permiten la manipulación independiente de dos parámetros vinculados a la presentación de estímulos: $P$ y Ť. El parámetro $P$ está asociado a la probabilidad de ocurrencia de estímulos en un ciclo $\mathrm{T}$ determinado, relativo al número total de ciclos $T$ programados. Por su parte, el parámetro T̄ esta asociado a la disponibilidad temporal para la entrega o presentación de un estímulo dentro de cada ciclo T.

En estudios recientes en los que se manipuló $P$ utilizando programas temporales señalados se observaron los siguientes resultados: a) un aumento gradual en la frecuencia de respuesta conforme disminuyó el valor de P. Esta función se mantuvo hasta llegar a valores por debajo de 0.3 , en los que se observó un descenso abrupto del responder que pudo llegar hasta niveles de extinción (e.g. Carpio, González \& Ribes, 1986; Ribes \& Torres, 1996; Ribes, Torres \& Mayoral, 2000a, 2000b, 2002a; Torres, Ribes \& Mayoral, 2003); y b) frecuencias de respuesta más altas en el subciclo $t^{\Delta}$ que en subciclo $t^{D}$ cuando el valor de Ť se mantuvo constante en 0.5 y P se programó en valores de 1.0 y 0.0 para $t^{\mathrm{D}}$ y $\mathrm{t}^{\Delta}$, respectivamente (Ribes \& Torres, 1996, 1997; Ribes, Torres \& Mayoral, 2000a, 2000b y 2002a). Las frecuencias de respuesta se equiparan cuando P es equivalente entre los subciclos (Ribes, Torres \& Piña, 1999). 
En lo concerniente a Ť, no se han encontrado efectos sistemáticos en la frecuencia de respuesta asociados a la manipulación de este parámetro. En un estudio inicial, Ribes, Carpio, Pallares y Torres (1986) evaluaron los efectos de reducir $\breve{T}(1.0,0.5,0.3,0.1,0.05)$ en un programa temporal de $68 \mathrm{~s}$, manteniendo señalizado el periodo de $\mathrm{t}^{\mathrm{D}}$. Los resultados no mostraron cambios sistemáticos en la frecuencia de respuesta en el subciclo $t^{\mathrm{D}}$, independientemente de la disminución en su duración relativa. En otro estudio, Ribes, Torres y Mayoral (2002b) manipularon el valor de Tॅ en un ciclo de 30 $\mathrm{s}$, manteniendo constante el valor de $\mathrm{P}$ bajo distintas condiciones de señalización de los subciclos $t^{\mathrm{D}}$ y $\mathrm{t}^{\Delta}$. Los resultados de este estudio fueron consistentes con los hallazgos reportados por Ribes et al. (1986) relativos a la constancia de la frecuencia de respuesta independientemente del valor de Ť. Sin embargo, se observó que bajo condiciones de señalización del subciclo $t^{D}$ hubo un aumento en la frecuencia de respuesta como función de la disminución de Ť hasta el valor de 0.01 , en el que la frecuencia descendió hasta valores cercanos a cero.

En un estudio reciente, Torres, Mayoral y Ribes (2008) evaluaron los efectos de manipular el valor de Ť bajo distintas condiciones de señalización de los subciclos en un programa temporal cuya duración del ciclo T fue de 60 $\mathrm{s}$, manteniendo el valor de $\mathrm{P}$ constante en 1.0. Aunque los datos no fueron concluyentes, se observó que dos de las cuatro ratas que se utilizaron en el estudio mostraron un incremento en la frecuencia de respuesta en el subciclo $t^{\mathrm{D}}$ conforme disminuía el valor de Ť hasta llegar al valor de 0.01 , en el que se observaron frecuencias de respuesta cercanas a cero. Las otras dos ratas no mostraron efecto alguno de la manipulación. Estos resultados parecen sugerir que existe una interacción entre la duración del ciclo $T$ y las funciones de respuesta obtenidas al manipular el valor de Tَ y de P. Por lo que el presente experimento tiene por objetivo evaluar los efectos de variar la disponibilidad y la probabilidad de entrega de agua bajo programas temporales no señalados de distinta duración.

\section{MÉTODO}

Sujetos

Se utilizaron 12 ratas albinas macho (cepa Wistar) de cinco meses de edad, experimentalmente ingenuas y cuyo peso ad libitum estuvo en un rango entre los 300 y los 450 gramos. Las ratas permanecieron alojadas en jaulas plásticas individuales, con acceso diario a agua purificada después de cada sesión experimental durante 60 min. Las ratas fueron privadas de agua 22.5 horas diarias antes del inicio del experimento. Las sesiones experimentales 
tuvieron lugar diariamente de $10: 00$ a $12: 00$ y de $16: 00$ a 17:00 horas, seis días de la semana durante once meses.

\section{Aparatos}

Se emplearon cuatro cámaras de condicionamiento operante de construcción propia (30 cm de largo por $25.5 \mathrm{~cm}$ de ancho y $32 \mathrm{~cm}$ de altura) colocadas dentro de cubículos sonoamortiguadores con extractores de aire de $115 \mathrm{w}$. Las cámaras tenían un dispensador de agua de $(0.01 \mathrm{cc})$ en la parte central inferior de la pared operativa, así como una palanca al lado izquierdo del dispensador de agua. Esta palanca se ubicó a $2.8 \mathrm{~cm}$ de la pared izquierda y a $2.5 \mathrm{~cm}$ encima del piso de rejilla. La palanca requirió de $0.13 \mathrm{~N}$ de fuerza para operar. Se utilizaron dos focos de $28 \mathrm{v}$. Un foco proporcionó la iluminación general de la cámara y se ubicó en la parte superior de la pared operativa, arriba del dispensador. Un segundo foco se ubicó dentro del dispensador de agua. La luz general de la cámara estaba encendida todo el tiempo excepto durante los $3 \mathrm{~s}$ del periodo de entrega de agua, en el que se encendía la luz del dispensador.

\section{Procedimiento}

Cada una de las ratas fue entrenada a presionar la palanca por moldeamiento directo, el cual concluyó cuando el animal obtuvo 100 entregas de agua consecutivas en la sesión. Una vez concluido el moldeamiento, las ratas se dividieron en tres grupos experimentales. Posteriormente se expusieron a un programa temporal de estímulos (Schoenfeld \& Cole, 1972). Cada uno de los grupos se expuso a duraciones del ciclo $T$ distintos. El Grupo 1 se expuso a un valor de ciclo T de $30 \mathrm{~s}$. El Grupo 2 a un valor de ciclo T de $120 \mathrm{~s}$. Por último el Grupo 3 se expuso a un valor de ciclo T de $360 \mathrm{~s}$.

Los grupos se sometieron a tres fases experimentales en las que se manipuló de manera descendente el valor de $\mathrm{P}$ utilizando los siguientes valores: $1.0,0.5,0.1$ (véase tabla 1). Cada una de las fases experimentales estuvo constituida por cuatro bloques de 20 sesiones. En cada bloque Tั tuvo un valor distinto. Los valores sucesivos de Ť fueron $1.0,0.5,0.3$ y 0.1 y se obtuvieron al variar la duración del subciclo $t^{\mathrm{D}}$ respecto del valor total de $\mathrm{T}\left(\mathrm{T}^{\circ}=\mathrm{t}^{\mathrm{D}} /\right.$ $T)$. En cada sesión, el ciclo $T$ iniciaba siempre con el subciclo $t^{D}$ y terminaba con el subciclo $t^{\Delta}$, repitiéndose la secuencia hasta concluir la sesión de una hora. En cada una de las sesiones, la entrega de agua fue contingente a la primera respuesta en el subciclo $t^{D}$ y correlacionó con el cese de la iluminación general de la caja y el encendido de la iluminación del bebedero. Si no ocurrían respuestas en el subciclo $t^{\mathrm{D}}$, la duración del ciclo T continuaba hasta el inicio del siguiente ciclo. No se programaron señales diferenciales asociadas a ninguno de los subciclos. 
Tabla 1. Valores de $P$ en cada una de las fases y valores de Ť en cada uno de los bloques para los tres grupos experimentales

\begin{tabular}{|c|c|c|c|c|c|c|c|c|c|c|c|c|}
\hline & \multicolumn{4}{|c|}{ FASE 1} & \multicolumn{4}{|c|}{ FASE 2} & \multicolumn{4}{|c|}{ FASE 3} \\
\hline $\begin{array}{c}\text { GRUPO } 1 \\
\mathrm{~T}=30^{\prime \prime} \\
\mathrm{n}=4\end{array}$ & \multicolumn{4}{|c|}{$p=1.0$} & \multicolumn{4}{|c|}{$p=0.5$} & \multicolumn{4}{|c|}{$p=0.1$} \\
\hline $\begin{array}{c}\text { GRUPO } 2 \\
\mathrm{~T}=120^{\prime \prime} \\
\mathrm{n}=4\end{array}$ & \multicolumn{4}{|c|}{$p=1.0$} & \multicolumn{4}{|c|}{$p=0.5$} & \multicolumn{4}{|c|}{$p=0.1$} \\
\hline $\begin{array}{c}\text { GRUPO } 3 \\
\mathrm{~T}=360^{\prime \prime}\end{array}$ & \multicolumn{4}{|c|}{$p=1.0$} & \multicolumn{4}{|c|}{$p=0.5$} & \multicolumn{4}{|c|}{$p=0.1$} \\
\hline $\begin{array}{c}\text { VALOR DE } \\
\text { T } \\
\end{array}$ & 1.0 & 0.5 & 0.3 & 0.1 & 1.0 & 0.5 & 0.3 & 0.1 & 1.0 & 0.5 & 0.3 & 0.1 \\
\hline SESIONES & 20 & 20 & 20 & 20 & 20 & 20 & 20 & 20 & 20 & 20 & 20 & 20 \\
\hline
\end{tabular}

\section{Resultados}

Los resultados se calcularon al obtener el valor mediano de respuestas por minuto (rpm) y de porcentaje de entregas de agua registradas para cada rata en cada una de las fases experimentales. Debido a que los datos mostraron ser consistentes entre sujetos, sólo se muestran los valores obtenidos por grupo.

La figura $1 \mathrm{~A}$ muestra la frecuencia mediana de rpm en los subciclos $t^{\mathrm{D}}$ $\mathrm{y} \mathrm{t}^{\Delta}{ }_{\left({ }_{11}\right)}$ y el porcentaje de entregas de agua obtenidas ${ }_{\left(Y_{2}\right)}$ como una función del valor de Ť en cada una de las fases experimentales para el Grupo 1. Se obtuvieron valores por debajo de la unidad, por lo que se representaron en un gráfico $\log _{10}$ con el fin de hacer más claras las diferencias de ejecución entre las condiciones. Los resultados mostraron un nivel de respuesta diferencial en cada una de las fases experimentales, así como un bajo porcentaje de entregas de agua obtenidas. No se observaron diferencias sistemáticas de respuesta entre los subciclos $t^{D}$ y $t^{\Delta}$. Durante la Fase $1(P=1.0)$ el rango de respuestas varió entre 1.0 y $0.6 \mathrm{rpm}$, observándose una función descendente de la ejecución conforme disminuyó el valor de Ť. Durante la Fase $2(P=0.5)$ se observó un incremento en el número de rpm en toda la fase (mayor a 1 rpm), con excepción del último bloque (广 $=0.1$ ) en el que se observó un nivel de respuestas de $0.3 \mathrm{rpm}$, por debajo del obtenido en la fase previa. Por su parte, durante la Fase $3(P=0.1)$ se observaron los valores más bajos de respuesta (entre 0.2 y $0.3 \mathrm{rpm}$ ). En este grupo se observó un bajo porcentaje de entregas de agua obtenidas por sesión (entre $30 \%$ y $2 \%$ ). El valor mediano del porcentaje de entregas de agua obtenido mostró una función descendente asociado al valor de Ť en las primeras dos fases. Durante la Fase 3, éste valor no fue mayor a $10 \%$ en $\breve{T}=1.0$, llegando a valores de cero a partir de $\check{T}=0.5$. 


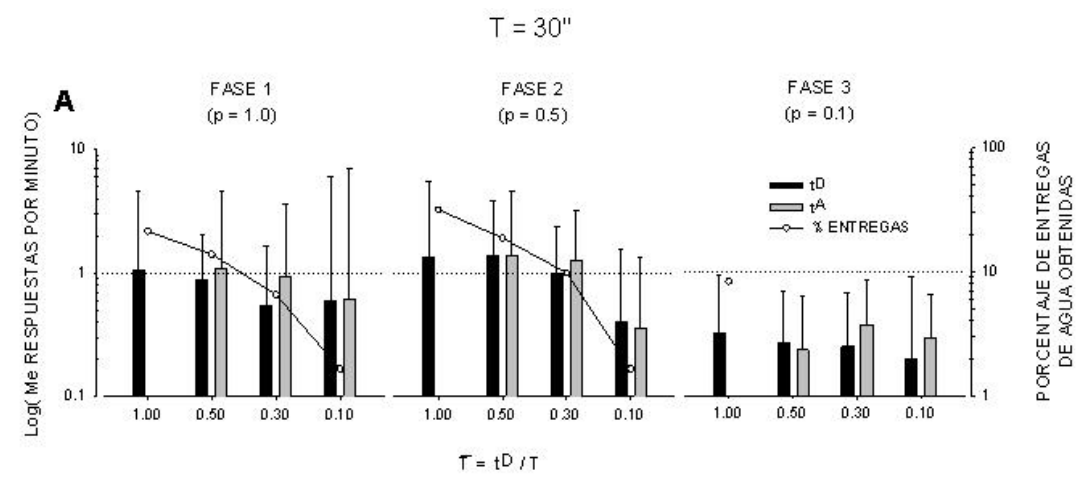

B

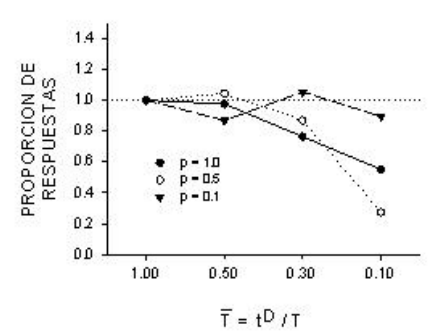

C

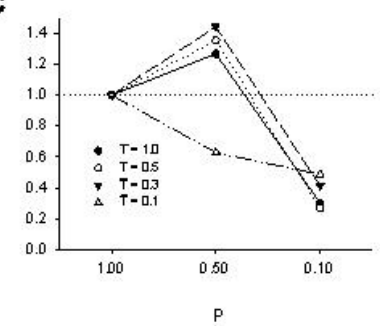

Figura 1. Frecuencia mediana de respuestas por minuto y porcentaje de entregas de agua obtenidas como función de los valores de $\bar{T}$ y $P$ en cada una de las fases experimentales (A), así como las variaciones en la proporción de respuesta para cada valor de disponibilidad (B) y probabilidad de reforzamiento (C) para el Grupo 1.

La figura 1B muestra la variación en la proporción de respuestas en cada valor de $\mathrm{P}$ como una función del valor de Ť en cada bloque. La variación se calculó tomando como punto de referencia inicial el número de rpm obtenido en cada ciclo $T$ de cada rata en el primer bloque $(\check{T}=1.0)$. El gráfico muestra efectos distintos del valor de Tॅ en la frecuencia de rpm en cada valor de $P$. Bajo las condiciones de $P=1.0$ y 0.5 , se observó una disminución gradual en la proporción de la tasa de respuesta asociada al descenso en los valores de Tั. Por su parte, en la condición de $\mathrm{P}=0.1$ no se observó un efecto sistemático sobre las respuestas asociado a los cambios en los valores de $\check{T}$.

La figura 1C muestra la variación en la proporción de respuestas en cada valor de Ť como una función del valor de P. Dicha variación se calculó tomando como punto de referencia inicial el número de rpm obtenido en cada ciclo 
T de cada rata en la primera fase $(P=1.0)$. Los datos mostraron que, con excepción de $\breve{T}=0.1$, en el que se observó una disminución gradual en la proporción de respuestas conforme disminuyó el valor de $\mathrm{P}$, en el resto de los valores de Ť se observó una función ascendente-descendente en la proporción de respuestas a lo largo de los distintos valores de P.

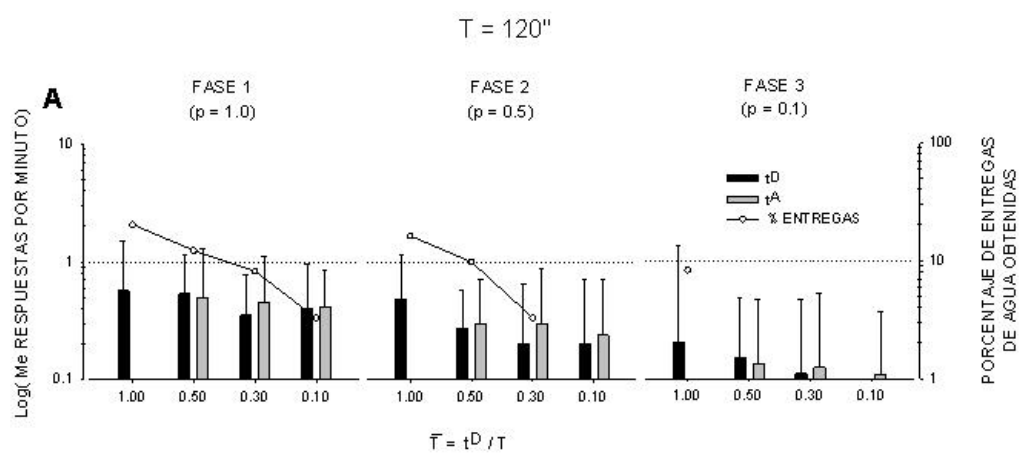

B

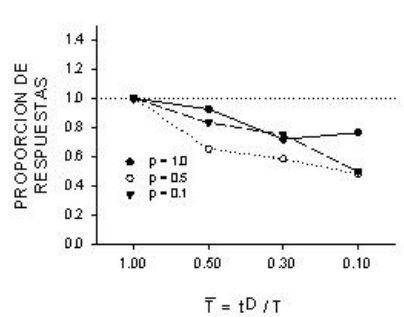

C

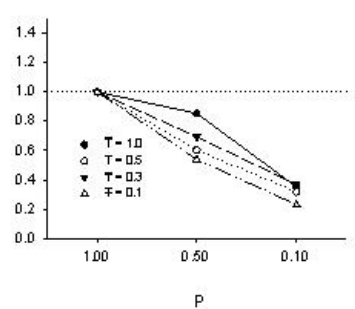

Figura 2. Frecuencia mediana de respuestas por minuto y porcentaje de entregas de agua obtenidas como función de los valores de $\bar{T}^{\prime}$ y $P$ en cada una de las fases experimentales (A), así como las variaciones en la proporción de respuesta para cada valor de disponibilidad (B) y probabilidad de reforzamiento (C) para el Grupo 2.

La figura 2A muestra la frecuencia mediana de rpm en los subciclos $t^{D}$ y $t^{\Delta}$ (Y1) y el porcentaje de entregas de agua obtenidas ${ }_{(\mathrm{Y} 2)}$ como una función del valor de T̄ en cada una de las fases experimentales para el Grupo 2. Los resultados mostraron un nivel por debajo de una rpm en las tres fases experimentales, así como un porcentaje muy bajo de entregas de agua obtenidas. Durante la Fase $1(P=1.0)$ el rango de respuestas estuvo entre 0.4 y $0.6 \mathrm{rpm}$, obteniéndose los niveles más bajos de ejecución en los valores de $\bar{T}=0.3$ 
y 0.1. No se observaron diferencias entre los subciclos $t^{\mathrm{D}}$ y $\mathrm{t}^{\mathrm{L}}$. El porcentaje de entregas de agua obtenidas se observó por debajo de $30 \%$ en $\check{T}=1.0$ y disminuyó gradualmente conforme fue descendiendo el valor de $\check{T}$. En la Fase $2(P=0.5)$ también se mostraron rangos de respuesta por debajo de una rpm. Durante el primer bloque ( $(\breve{T}=1.0)$ el valor mediano de rpm fue de 0.6 y fue descendiendo hasta llegar a valores cercanos a $0.2 \mathrm{rpm}$ en $\check{T}=0.3 \mathrm{y}$ 0.1 . El porcentaje de entregas de agua obtenidas fue menor a $30 \%$ y mostró una función descendente asociada a los decrementos en el valor de Ť. Por último, en la Fase $3(P=0.1)$ se observó una caída en el nivel de ejecución (por debajo de $0.2 \mathrm{rpm}$ ) a lo largo de los cuatro bloques que conformaron la fase, llegando a valores cercanos a la extinción en $\bar{T}=0.1$. El porcentaje de entregas de agua obtenidas estuvo en valores menores a $10 \%$ durante la condición de $\check{T}=1.0$. En el resto de los bloques el valor mediano del porcentaje fue de 0 .

La figura 2B muestra la variación en la proporción de respuestas en cada valor de $\mathrm{P}$ como una función del valor de Ť. Dicha variación se calculó tomando como punto de referencia inicial el número de rpm obtenido en cada ciclo T de cada rata en el primer bloque $(\check{T}=1.0)$. El gráfico muestra que independientemente del valor de P, la disminución del valor de Ť tuvo un efecto de decremento en la proporción de respuestas.

La figura $2 \mathrm{C}$ muestra la variación en la proporción de respuestas en cada valor de T̄ como una función del valor de P. Dicha variación se calculó tomando como punto de referencia inicial el número de rpm obtenido en cada ciclo $T$ de cada rata en la primera fase $(P=1.0)$. En la figura se muestra que la disminución en los valores de $\mathrm{P}$ tuvo por efecto la disminución en la proporción de respuesta, independientemente del valor de $\check{T}$. 


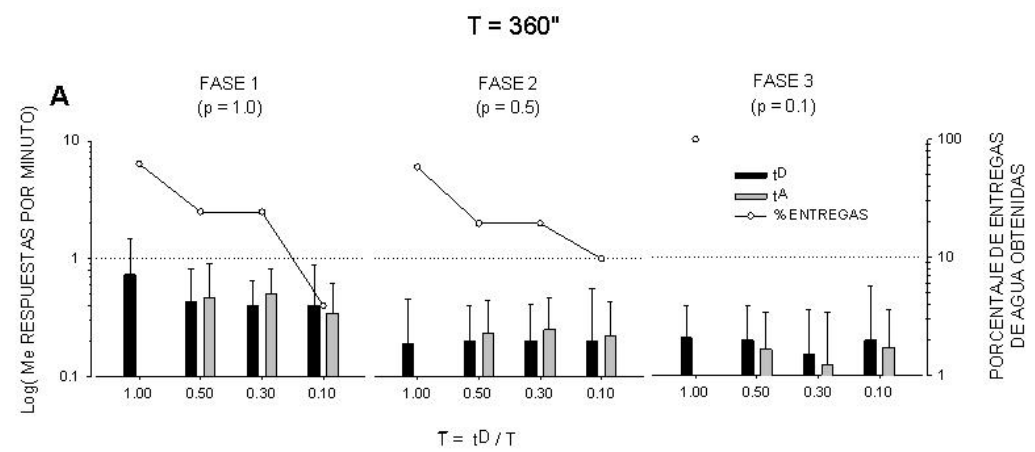

B

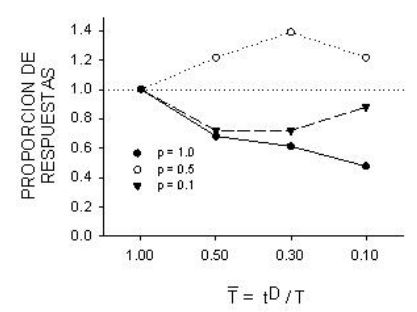

C

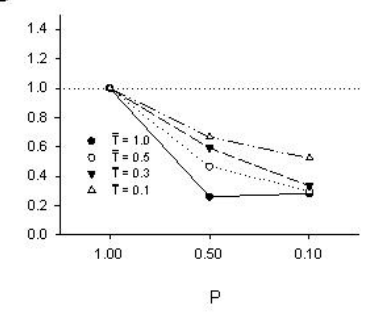

Figura 3. Frecuencia mediana de respuestas por minuto y porcentaje de entregas de agua obtenidas como función de los valores de $\dot{T}$ y $P$ en cada una de las fases experimentales (A), así como las variaciones en la proporción de respuesta para cada valor de disponibilidad (B) y probabilidad de reforzamiento (C) para el Grupo 3.

La figura $3 A$ muestra la frecuencia mediana de rpm en los subciclos $t^{D}$ y $t^{\Delta}{ }_{(Y 1)}$ y el porcentaje de entregas de agua obtenidas ${ }_{\left(Y_{2}\right)}$ como una función del valor de $T^{\circ}$ en cada una de las fases experimentales para el Grupo 3. En general se observó un nivel bajo de respuestas, así como una función descendente a lo largo de las fases experimentales. No se observaron efectos locales en los subciclos $t^{D}$ y $t^{\Delta}$. Durante la primera fase $(P=1.0)$ se observó un rango entre 0.7 a $0.4 \mathrm{rpm}$ tanto en $\mathrm{t}^{\mathrm{D}}$ como en $\mathrm{t}^{\mathrm{L}}$. La frecuencia más alta de respuestas se observó durante $\check{T}=1.0(0.7 \mathrm{rpm})$. En el resto de los valores de ${ }^{\top}$ la tasa de respuesta se mantuvo en valores cercanos a $0.4 \mathrm{rpm}$. Se observó también una frecuencia de rpm ligeramente mayor en el subciclo $t^{\Delta}$, con excepción del bloque 4 ( $(\bar{T}=0.1)$. Durante la Fase $2(P=0.5)$ no se observaron cambios sistemáticos en la frecuencia de rpm en ninguno de los bloques, manteniéndose en valores cercanos a $0.2 \mathrm{rpm}$ tanto en $\mathrm{t}^{\mathrm{D}}$ como en $\mathrm{t}^{\Delta}$. Este nivel de 
respuestas se mantuvo durante la Fase $3(P=0.1)$ con excepción del bloque 3 ( $\breve{T}=0.3$ ), en el que se observó una disminución de las rpm hasta valores cercanos a 0.1. El porcentaje mediano de entregas de agua obtenidas mostró una función descendente a lo largo de los bloques en las dos primeras fases $(P=1.0$ y 0.5$)$, variando de $70 \%$ en $\check{T}=1.0$ a valores por debajo de $10 \%$ en $\check{T}=0.1$. Durante la Fase $3(P=0.1)$, se observó que durante el primer bloque ( $\check{T}=1.0$ ) el porcentaje mediano de entregas de agua obtenidas fue de $100 \%$. Posteriormente dicho porcentaje disminuyó hasta valores de cero en el resto de los bloques.

La figura 3B muestra la variación en la proporción de respuestas en cada valor de $\mathrm{P}$ como una función del valor de Ť. Dicha variación se calculó tomando como punto de referencia inicial el número de rpm obtenido en cada ciclo T por cada rata en el primer bloque ( $(\breve{T}=1.0)$. Bajo la condición de $P=1.0$ se observó una disminución gradual en la frecuencia de respuesta asociada a una disminución del valor de Ť. Bajo la condición de $P=0.5$, se observó un incremento en el nivel de respuesta conforme Ť disminuyó. Este efecto se observó hasta el valor de $\Psi^{\top}=0.1$, en el que el nivel de respuesta disminuyó. Por su parte, en la condición de $P=0.1$ se observó una disminución gradual de las respuestas conforme el valor de Ṫ disminuyó, observándose un ligero incremento en el valor de $\check{T}=0.1$.

La figura $3 \mathrm{C}$ muestra la variación en la proporción de respuestas en cada valor de Ť como una función del valor de P. Dicha variación se calculó tomando como punto de referencia inicial el número de rpm obtenido en cada ciclo T de cada rata en la primera fase $(P=1.0)$. Los datos muestran que independiente mente del valor de Ť, se observó una función descendente en el nivel de respuestas conforme disminuyó el valor de P. Es importante señalar que el efecto más pronunciado se observó cuando $\mathrm{T}=1.0$.

\section{DISCUSIÓN}

Los resultados de este estudio confirman la naturaleza funcionalmente independiente de los parámetros $\mathrm{P}$ y $\breve{T}$. Sin embargo, al parecer las funciones de estos parámetros dependen de la duración absoluta del ciclo T. En el Grupo $1(T=30)$ se pudieron replicar las funciones de respuesta asociadas a la manipulación de P. En este grupo se observó un incremento en la frecuencia de respuesta asociado al descenso del valor de $\mathrm{P}$ de $1.0(1.0 \mathrm{rpm})$ a 0.5 (1.4 $\mathrm{rpm}$ ), para posteriormente observar un decremento en la frecuencia de respuesta hasta valores por debajo de los $0.3 \mathrm{rpm}$ cuando $P$ tuvo un valor de 0.1 . Estos resultados son consistentes con los obtenidos por Carpio et al. (1986) y Ribes y Torres (1996). Sin embargo, en los Grupos 2 y 3 ( $T=120$ y $T=360$, respectivamente) la frecuencia de respuesta mostró una función descenden- 
te (de alrededor de $1 \mathrm{rpm}$ a $0.2 \mathrm{rpm}$ ), asociada al decremento de los valores de P. Estos resultados confirman el carácter relativo del parámetro, en la medida que los efectos de la reducción de $\mathrm{P}$ al parecer dependen del valor absoluto del ciclo $\mathrm{T}$, más que de los valores paramétricos particulares.

Cabe mencionar que el valor mediano de la frecuencia de rpm en este estudio fue baja respecto de programas temporales que utilizan estímulos asociados (Ribes, Torres \& Mayoral, 2002; Torres, Mayoral \& Ribes, 2008), en los que se llegaron a observar ejecuciones por arriba de $5 \mathrm{rpm}$. Este efecto puede deberse, tal y como lo sugirieron Ribes y Torres (1996) y Ribes, Torres, Barrera y Mayoral (1997), a que los estímulos asociados a los subciclos adquieren funciones de "preparador (priming)", auspiciando el responder en cada uno de los ciclos. Ahora bien, independientemente de la frecuencia de respuesta observada, el porcentaje de entregas de agua fue similar entre estos estudios, lo que nos habla de la importancia de la distribución temporal de la respuesta bajo este tipo de contingencias. En estudios previos (Ribes et al., 2000a; Ribes et al., 2007), se ha encontrado que la frecuencia de respuesta en la sesión no afecta necesariamente el porcentaje de entregas de agua. Esto parece sugerir que la distribución del responder en cada ciclo T determina la obtención o pérdida del reforzador, lo que implica que dos distribuciones locales diferentes de una misma frecuencia de respuestas por sesión determina porcentajes distintos de entregas de agua obtenidas, posiblemente debido a que las respuestas pueden ocurrir en el remanente de $t^{D}$ una vez entregada el agua, o pueden ocurrir en $t^{\Delta}$ en donde $P=0.0$.

Una de las características primordiales de los programas temporales es la disponibilidad limitada del reforzador dentro del ciclo T. En la medida en que no ocurra una respuesta dentro del periodo particular de disponibilidad $\left(\mathrm{t}^{\mathrm{D}}\right)$ se pierde la entrega programada, a diferencia de los programas de reforzamiento tradicionales (Ferster \& Skinner, 1957) en los que el programa "espera" la respuesta para entregar el reforzador. Es decir, una vez que se ha alcanzado el requisito para que la respuesta sea reforzada (valor del intervalo en los programas basados en tiempo y el número de respuestas requeridas menos una en los programas de razón), el programa no inicia una nueva contingencia hasta que se presente la respuesta criterio para la entrega del reforzador. Esto hace que una vez disponible, el reforzador no se "pierda".

A partir de esta consideración, se puede suponer que la variabilidad obtenida en este estudio puede deberse a la variabilidad temporal en la entrega del agua, propia de la interacción entre la aleatoriedad de la probabilidad de reforzamiento y la ausencia de respuestas en los ciclos con disponibilidad y la duración de cada uno de los subciclos particulares. En este sentido, los programas temporales requieren de tasas o frecuencias diferenciales de respuesta para que ocurra el reforzamiento, a diferencia de los programas tradicionales en donde no se prescriben periodos críticos para la emisión de 
respuesta y la consecuente entrega del reforzador (Ferster \& Skinner, 1957; Lee, Sturmey \& Fields, 2007).

A diferencia de lo propuesto por Martin (1971), estos datos dan soporte al hecho de reconocer que la diferencia entre $\mathrm{P}$ y Tั tiene que ver con la distribución temporal versus la disponibilidad temporal en la presentación de los estímulos. El parámetro $\mathrm{P}$ hace referencia a la distribución temporal de la entrega de agua durante la sesión y se sintetiza en términos de la razón $\mathrm{T} / \mathrm{P}$, mientras que el parámetro de Ť se relaciona directamente con el valor absoluto del tiempo disponible para responder y que se presente el agua, independientemente de la frecuencia programada. La disponibilidad limitada dentro y entre ciclos de reforzamiento evidentemente afecta la "consistencia" en la ejecución que caracteriza el método de la operante libre.

\section{REFERENCIAS}

Carpio, C. A., González, R. \& Ribes, E. (1986). Probabilidad de reforzamiento y su señalización en un programa definido temporalmente. Revista Mexicana de Análisis de la Conducta, 12, 89-104.

Ferster, C. B. \& Skinner, B. F. (1957). Schedules of reinforcement. Nueva York: Appleton Century Crofts.

Lee, R., Sturmey, P. \& Fields, L. (2007). Schedule-induced and operant mechanisms that influence response variability: a review and implications for future investigations. The Psychological Record, 57, 429-455.

Martin, J. M. (1971). Temporally defined schedules of stimulus correlations. Tesis doctoral inédita. City University of New York.

Neuringer A. J. \& Chung, S. (1967). Quasi-reinforcement: control of responding by a percentage-reinforcement schedules. Journal of the Experimental Analysis of Behavior, 10, 45-54.

Ribes, E., Carpio, C. A., Pallares, A. \& Torres, J. (1986). Efectos de la reducción en la disponibiliad de reforzamiento en un programa temporal señalado. Revista Mexicana de Análisis de la Conducta, 22, 127-136.

Ribes, E. \& Torres, C. (1996). Efectos de la variación de la probabilidad de reforzamiento correlacionada con dos estímulos neutros en un programa definido temporalmente. Revista Mexicana de Análisis de la Conducta, 22, 41-78.

Ribes, E. \& Torres, C. (1997). Stimulus functions: lack of discrimination or faluire of generalization? Revista Mexicana de Análisis de la Conducta, 23, 249-274.

Ribes, E., Torres, C. \& Piña, J. (1999). Comparación de los efectos de la presencia y ausencia de estímulos diferenciales en dos programas temporales con probabilidades variantes igualadas. Acta Comportamentalia, 7, 5-29.

Ribes, E., Torres, C. \& Mayoral, A. (2000 a). Señalización no diferencial de distintas probabilidades de entrega de agua en dos subciclos de un programa definido temporalmente. Acta Comportamentalia, 8, 5-21. 
Ribes, E., Torres, C. \& Mayoral, A. (2000 b). Efectos de la ausencia y presencia de estímulos correlacionados con distintas probabilidades de reforzamiento y con extinción en programas definidos temporalmente. Revista Mexicana de Análisis de la Conducta, 26, 327-354.

Ribes, E., Torres, C. \& Mayoral, A. (2002 a). Extended exposure to a discriminated limited-hold temporal schedule does not produce stimulus control. Behavioural Processes, 59, 131-146.

Ribes, E., Torres, C. \& Mayoral, A. (2002 b). Señalización de la entrega y no entrega de agua en programas temporales con variaciones en la disponibilidad de reforzamiento. Acta Comportamentalia, 10, 129-149.

Ribes, E., Zepeda, I., Arenas, S. \& Mayoral, A. (2007). Efecto de la densidad local de entrega de agua en la frecuencia total de respuesta en un programa temporal señalado. Revista Mexicana de Análisis de la Conducta, 33, 139-166.

Schoenfeld, W. N. \& Cole, B. K. (1972). Stimulus schedules: the t-tau system. New York: Harper \& Row Publishers.

Schoenfeld, W. N., Cumming, W. W. \& Hearst, E. (1956). On the classification of reinforcement schedules. Proceedings of the National Academy of Science, 42, 536570 .

Staddon, J. E. R. \& Innis, N. K. (1969). Reinforcement omission on fixed interval schedules. Journal of the Experimental Analysis of Behavior, 12, 689-700.

Skinner, B. F. (1938). The behavior of organisms. Nueva York: Appleton Century Crofts.

Torres, C., Mayoral, A. \& Ribes, E. (2008). Análisis de la variación en la disponibilidad en la entrega de agua bajo distintas condiciones de señalización de los subciclos en un programa temporal. Universitas Psychologica, 7, 535-547.

Torres, C., Ribes, E., \& Mayoral, A. (2003). Efectos de probabilidades complementarias y contrastadas de reforzamiento con y sin señalización en programas temporales. Acta Comportamentalia, 11, 130-149. 